\title{
Ball's Motion Estimation Using a Line-Scan Camera
}

\author{
Zhuxin Zhao*, Gongjian Wen \\ ATR Key Lab., School of Electronic Science and Engineering, National Univ. of Defense Technology, Changsha, China \\ *zzxwan2000@gmail.com
}

\begin{abstract}
An original method is proposed for estimating the position and velocity of a moving ball using a single line-scan camera. The method consists of two steps: firstly, the ball's initial position and velocity are determined by several key points in the image, and secondly, these parameters are further refined by a model-based optimization algorithm. The algorithm achieves accurate parameter estimation by matching the projection contour of the real ball and that of the same size sphere model. Finally, the relevance and accuracy of the method are experimentally confirmed on synthetic and real images.
\end{abstract}

Keywords: Position, velocity, ball motion estimation, line-scan camera

\section{INTRODUCTION}

$\mathrm{T}$ HE ESTIMATION of a ball's 3D motion, such as position and velocity, is an interesting question in sport event analysis. There have been many researches on this issue. The approach based on image sequence always requires quite costly vision systems to make the ball visible in multiple synchronized cameras [1]. In recent years, several techniques have been developed to deal with the motion of a flying ball using a single image [2-5]. Most of these studies focus on the motion blur induced by the moving ball's position change during the exposure time. Algorithms of image deblurring or ellipse extraction are introduced to obtain the motion extent, which always lead to complex image processing and feature extraction. Additionally, the motion details in depth direction are difficult obtain by these methods. It is noted that all these methods are based on images collected by area array sensors.

In our study, an attempt has been made to fulfill the task using a line-scan camera. This kind of camera uses one linear array sensor to capture a two-dimensional image by composing lines collected at different time instants. Currently, the line-scan camera has been only used for automatic inspection $[7,8]$. We believe that, actually, the potential of the camera is underestimated. The line-scan camera has several unique characteristics. Firstly, it is much more cost-effective. Only one linear array sensor is used, which means it can provide higher scanning frequency (up to $140 \mathrm{kHz}$ ). Secondly, the background of the images collected is uniform when the camera is stationary. Therefore, the camera can record a clear moving object with very sharp contour. However, few works have focused on the value of the line-scan camera in motion analysis $[8,9]$.

In this article, an original method is proposed for estimating motion parameters of a ball from the image collected by a single line-scan camera. In section 2, the projection model of the camera is described. The method consists of two steps: firstly, the initial value of position and velocity of the moving ball is determined, and then these 3D motion parameters are further refined by a model-based optimization algorithm. In section 3 , experiments are made to testify the presented method using synthetic and real data.

\section{METHODS}

The measurement work is accomplished by a calibrated line-scan camera. As a measure camera rather than an inspection tool, the parameters of the line-scan camera are necessary. This is related to the calibration and projection model of the line-scan camera which is well analyzed in literature $[10,11]$. The procedure of the calibration is not detailed in this article, but it is necessary when real images are collected. The projection model is described as below.

\section{A. Projection Model}

Only one line-scan camera is used to capture the image of the flying ball. Three coordinate systems: the object space (world) coordinate $O-X Y Z$, the image space coordinate $o-x y z$ and 2D image plane coordinate $o^{\prime}-x^{\prime} y^{\prime}$ are used (Fig.1(a)). The image space coordinate takes the projective center of the camera as its origin point. The direction of the $z$ axis points to the primary optical axis. The $x$ and $y$ axes are vertical and parallel with the linear array sensor, respectively. The image plane coordinate is on the plane $z=-f$ ( $f$ is the focal length). The origin point $o^{\prime}$ is the principle point, and the $x^{\prime}$ and $y^{\prime}$ axes are parallel with the $x$ and $y$ axes, respectively.

The projection model serves to describe the relationship between a point in the object space coordinate and its projection on the image. It is related to the elements of exterior and interior orientation factors, which are determined in a camera calibration procedure. Only the points on the viewing plane (Fig.1) can be projected, that is to say, $x=x^{\prime}=0$, so an arbitrary space point $(X, Y, Z)$ on the viewing plane could be projected at $\left(0, y^{\prime}\right)$ by the projection model [11]:

$$
\left\{\begin{array}{l}
y^{\prime}=\frac{n_{1} Y+n_{2} Z+n_{3}}{n_{4} Y+n_{5} Z+1} \\
X=p Y+q Z+r
\end{array}\right.
$$

where $n_{1}, n_{2}, n_{3}, n_{4}, n_{5}, p, q, r$ are related to the elements of 
the camera. If we take the image space coordinate as the measurement coordinate, Eq. (1) can be simply written as

$$
\left\{\begin{array}{l}
x^{\prime}=0 \\
y^{\prime}=-\frac{y}{z} f
\end{array}\right.
$$

Eq. (2) is a simpler model. In real measurement procedure, the position and velocity are always computed in image space coordinate, and then transformed into object space coordinate if necessary.

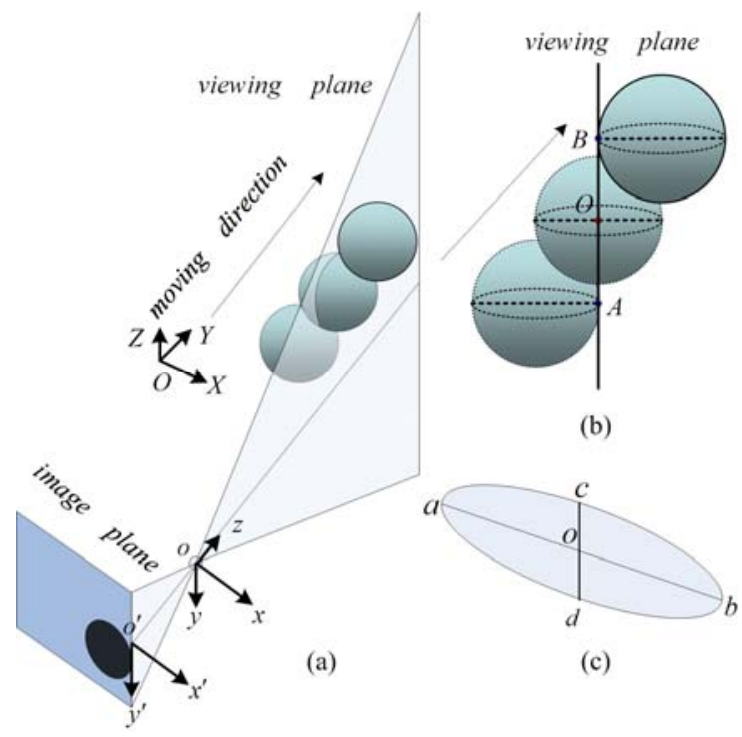

Fig.1. (a) The coordinate systems and the moving ball; (b) the ball flying across the viewing plane; (c) the linear image of the moving ball.

\section{B. Initial Guess}

If the projection model is given, every image point can establish the imagery relationship with its space point. In this section, an initial guess of the ball's motion parameters is computed using four key points [12]. As shown in Fig.1(a), a ball moves across the viewing plane of the line-scan camera. Fig.1(b) shows the details of the ball flying across the viewing plane. The ball is firstly scanned by the viewing plane at point $A$ with its projection point $a$ on the image plane, and then the ball center $O$ moves across the viewing plane with its projection point $o$. When the last point $B$ is scanned with its projection point $b$, the whole ball image is collected, which is composed of plenty of line images. The linear array image of the ball always appears deformed because of the relative movement between the ball and the camera (Fig.1(c)). In Fig.1(c), $a, b$ are the first and last projection points, respectively, when the ball is scanned. The middle point of $a b$ on the image is $o$, the projection point of the ball center. The projection of the ball is a line segment when the ball center is scanned. Here we use $c, d$ to denote the two tips of the projective line segment.

The points $a, b, c, d$ are the key points picked from the image, which can be used to compute the initial guess of the position and velocity parameters.
Firstly, we compute the initial value of the position of the ball center when it is scanned by the viewing plane. Fig. 2 shows the cross sectional view when the ball center $O$ moves across the viewing plane.

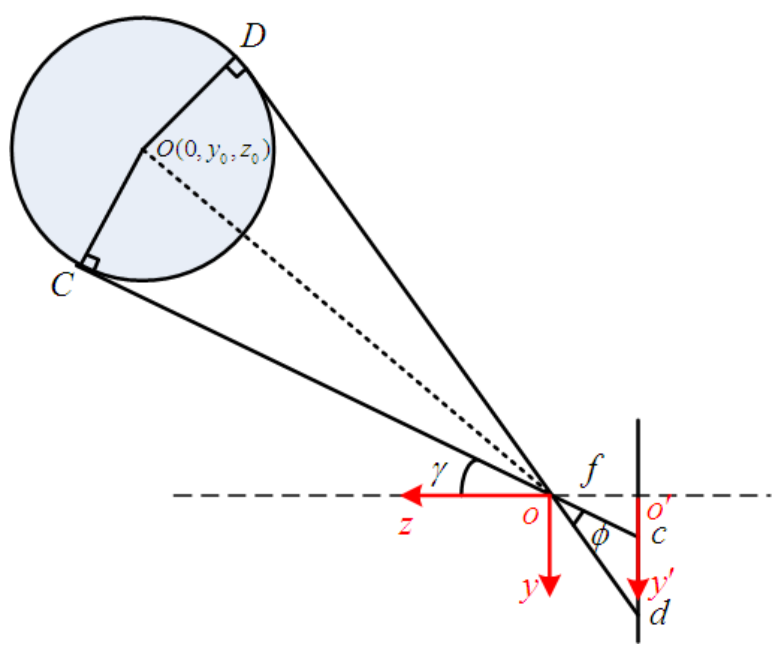

Fig.2. The cross sectional view of ball when the center of the ball is crossing the $o-y z$ plane.

Suppose the coordinate of the ball center $O$ at this time is $O\left(0, y_{0}, z_{0}\right)$. The projection of the circle cross section on the image plane is the line segment with two tips $c$ and $d$. Suppose points $C$ and $D$ are the correspondent space points on the ball whose projections on the image are points $c$ and $d$, respectively. Therefore, $C c$ and $D d$ are two tangent lines of the circle.

Let $\gamma$ be the angle between $C c$ and the $z$ axis, $\phi$ be the angle between $C c$ and $D d, y_{c}^{\prime}, y_{d}^{\prime}$ be the coordinate values of $c$ and $d$ on the $y^{\prime}$ axis, respectively, $f$ be the focus length of the line-scan camera. Then $\gamma=\arctan \left(y_{c}^{\prime} / f\right)$, $\phi=\arctan \left(y_{d}^{\prime} / f\right)-\arctan \left(y_{c}^{\prime} / f\right)$, and the values of $y_{0}, z_{0}$ are given by

$$
\begin{gathered}
y_{0}=-R \sin (\gamma+0.5 \phi) / \sin (0.5 \phi) \\
z_{0}=R \cos (\gamma+0.5 \phi) / \sin (0.5 \phi)
\end{gathered}
$$

Therefore, the position of the ball can be determined if the ball radius, coordinate values of $c$ and $d$, and the focus length are given.

We consider the details of points $A$ and $B$ crossing the viewing plane. Suppose the coordinate of point $A$ is $A\left(0, y_{1}, z_{1}\right)$, and the coordinate of point $B$ is $B\left(0, y_{2}, z_{2}\right)$, when they are scanned by the viewing plane, respectively. According to Eq. (2), we obtain the equations

$$
\left\{\begin{array}{l}
-\frac{y_{1}}{z_{1}} f=y_{a}^{\prime} \\
-\frac{y_{2}}{z_{2}} f=y_{b}^{\prime}
\end{array}\right.
$$


Where $f$ is the focus length, and $y_{a}^{\prime}, y_{b}^{\prime}$ are the coordinate values of $a$ and $b$ on the $y^{\prime}$ axis, respectively.

Taking the motion and geometrical characteristics of the ball target into consideration, we can also obtain the relationship

$$
\left\{\begin{array}{l}
y_{1}+y_{2}=2 y_{0} \\
z_{1}+z_{2}=2 z_{0}
\end{array}\right.
$$

The values of $y_{1}, y_{2}, z_{1}, z_{2}$ can be computed from equations (5) and (6).

Time $\mathrm{T}$, necessary for the ball to fly across the viewing plane, can be determined by computing the number of pixels that covers from $a$ to $b$ along the $x^{\prime}$ axis. Radius $R$ of the ball is known. Suppose the ball's velocity is $\mathbf{v}\left(v_{x}, v_{y}, v_{z}\right)$, then

$$
\left\{\begin{array}{l}
v_{x}=2 R / T \\
v_{y}=\left(y_{2}-y_{1}\right) / T \\
v_{z}=\left(z_{2}-z_{1}\right) / T
\end{array}\right.
$$

Therefore, an initial guess of the position and velocity of the moving ball are obtained.

\section{Model-Based Optimization Algorithm}

The accuracy of the initial guess depends on the accuracy of the key points used. In order to reduce the uncertainties caused in the pick-up of the key points, a model-based optimization algorithm is developed considering the whole contour information of the ball's image rather than only four points. The algorithm achieves accurate parameter estimation by matching the projection contour of the real ball with that of the same size sphere model. Therefore, the estimation of motion parameters is transformed into a problem of imagematching. Our task is to adjust the motion parameters of the dynamic model to reduce the difference between the simulated image and the real one. An optimization algorithm based on the gradient information of the real and simulated image is used to fulfill the task. The procedure flow is presented in Fig.3 (taking a moving basketball as example).

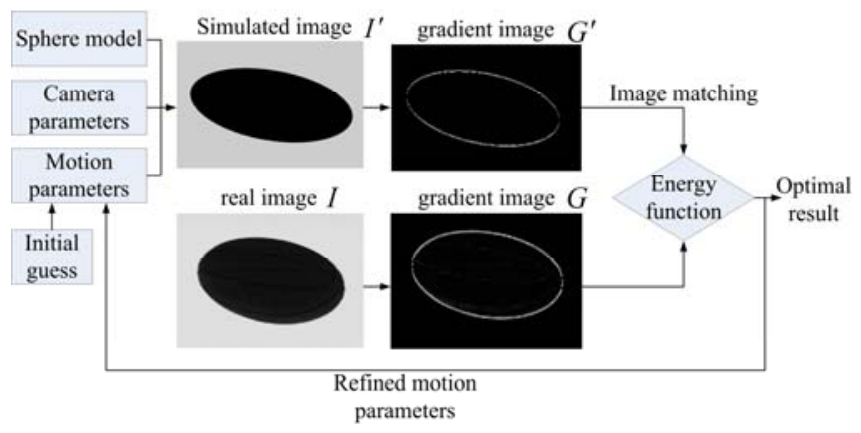

Fig.3. The procedure flow of the optimization algorithm.

\section{a. Dynamic sphere model}

The ball can be regarded as a moving geometrical sphere in the image space coordinate. Dynamic sphere model $S$ is established with respect to the diameter $R$, position of center point $\mathbf{p}\left(x_{0}, y_{0}, z_{0}\right)$, the velocity $\mathbf{v}\left(v_{x}, v_{y}, v_{z}\right)$ and time $t$ of the moving ball

$$
\begin{aligned}
S(\mathbf{p}, \mathbf{v}, t ; R)= & \left(x-x_{0}-v_{x} t\right)^{2}+\left(y-y_{0}-v_{y} t\right)^{2} \\
& +\left(z-z_{0}-v_{z} t\right)^{2}-R^{2}
\end{aligned}
$$

\section{b. Simulated image}

The highlight of this paper is the idea of image-matching for motion estimation. The simulated images, used for matching with the real image, are generated in Microsoft Visual C++ 6.0 program software. Given the dynamic sphere model, the camera parameters, the ball's position and velocity, the simulated theoretical images of the dynamic sphere model can be generated according to the projection model. The linear arrays make up the simulated image line by line with the time $t$. When the dynamic sphere model comes across the viewing plane, each linear array includes a line segment projected by the circle cross section. The continuous line segments form a deformed ball contour line by line until the ball passes the viewing plane. A simulated image is shown in Fig.3.

\section{c. Optimization Algorithm}

The motion parameter estimation is considered as a problem of image matching in this paper. That is to say, if the simulated and the real images are collected with the same ball size and the same camera parameters, their mutual match will be perfect only when the preset motion parameters of the simulated image are closest to the real ball's motion parameters.

In order to compare the simulated images with the real ones, we take the ball's contour feature into account. The gradient information is commonly used to describe the target contour feature. Because the line-scan camera is fixed, the background of the moving ball's linear array image is quite clear. Moreover, since the linear array image is assembled with scanning lines, the gradient magnitude is computed only on the direction of linear array, which is really different from the processing of the area array image.

Let $\left(x_{i}, y_{j}\right), i=1 \cdots N, j=1 \cdots M$ be the $N \times M$ pixels of real image $I$. Let the gradient image be $G$, then

$G\left(x_{i}, y_{j}\right)=\left|I\left(x_{i}, y_{j+1}\right)-I\left(x_{i}, y_{j}\right)\right|, i=1 \cdots N, j=1 \cdots M-1(7)$

Let $\left(x_{i}^{\prime}, y_{j}^{\prime}\right), i=1 \cdots N, j=1 \cdots M$ be the $N \times M$ pixels of theoretical image $I^{\prime}$ related to the dynamic sphere model $S(\mathbf{p}, \mathbf{v}, t ; R)$. Let the gradient image be $G^{\prime}$, then

$G^{\prime}\left(x_{i}^{\prime}, y_{j}^{\prime}\right)=\left|I\left(x_{i}^{\prime}, y_{j+1}^{\prime}\right)-I\left(x_{i}^{\prime}, y_{j}^{\prime}\right)\right|, i=1 \cdots N, j=1 \cdots M-1(8)$

A moving basketball's linear array image, the same size sphere model's simulated image, and their gradient image are 
shown in Fig.3. Our task is to adjust the motion parameters of the dynamic sphere model to minimize the difference between the contours of the theoretical simulated projection and that of the real one. An energy function based on the comparison of the gradient information is established by

$$
\varepsilon=\sum_{i=1}^{N} \sum_{j=1}^{M-1}\left[G^{\prime}\left(x_{i}^{\prime}, y_{j}^{\prime}\right)-G\left(x_{i}, y_{j}\right)\right]^{2}
$$

The value of the energy function indicates the similarity degree between the simulated and the real image. We want to find $\mathbf{p}, \mathbf{v}$ that could minimize the energy function. Therefore, the problem is actually a nonlinear optimization problem as indicated below

$$
\begin{aligned}
\min \varepsilon & =\min \left\{\sum_{i=1}^{N} \sum_{j=1}^{M-1}\left[G^{\prime}\left(x_{i}^{\prime}, y_{j}^{\prime}\right)-G\left(x_{i}, y_{j}\right)\right]^{2}\right\} \\
& =\min \left(\left\|g^{\prime}-g\right\|_{2}\right)
\end{aligned}
$$

Where $g^{\prime}, g$ are the vectors of the gradient images of the theoretically simulated and the real images, $g^{\prime}, g \in R_{+}^{N \times(M-1)}$. Powell optimization algorithm can be used to solve the multidimensional nonlinear optimization problem. The initial guess helps to start the optimization and converge to an optimal solution. When the algorithm reaches the optimal solution, the values of $\mathbf{p}, \mathbf{v}$ can be regarded as the most reasonable estimation of the real ball's motion parameters.

\section{EXPERIMENT}

The proposed method has been validated both with synthetic and real camera images. The preset motion parameters, before these images were collected, are regarded as ground truth. In this section, the effectiveness of the proposed method is tested, and we conduct error analysis by comparing the estimation results and their ground truth.

\section{A. Tests on Synthetic Data}

Synthetic images are collected using a digital ball instead of a real ball. The synthetic tests are necessary and helpful because the synthetic images can be produced theoretically when the ball motion parameters and camera parameters are given. It is easy to obtain the theoretical linear array image of a moving ball at any preset size, position or velocity.

i) The digital ball is established in 3D Max software platform (Fig.4(a)), and the digital ball data is exported in the 3ds file;

ii) The digital ball data is read and transformed into the object space coordinate;

iii) The projection model of the line-scan camera is programmed in Microsoft Visual C++ 6.0. When the space points on the digital ball move from preset position and in preset velocity, a synthetic linear array image is observed. Fig.4(b) (e) illustrate several of the synthetic images related to different preset motion parameters. The preset parameters provide ground truth to compare with the estimated results.
In order to test the effectiveness of the method, many synthetic images with different ball size, velocity, and camera scanning frequency are simulated. According to the proposed method, firstly, an initial guess of the ball motion is made. As shown in Fig.4(b), the first and last projection point $a$ and $b$ are picked, and then the scanning line produced when the ball center was scanned, is marked with a red-dotted line. The two intersection points produced by the red-dotted line and the ball contour are the two points $c$ and $d$, respectively. The initial guess is computed from the coordinate values of the 4 key points on the image. Secondly, a proposed model-based optimization algorithm is used to refine the initial guess by taking the whole ball contour information into consideration. In order to graphically depict the estimation results, we draw the simulated projection contours of both initial and optimal estimation results on the synthetic images. Theoretically, the projection contour will match with the ball image very well if the estimation result matches with the preset parameters. Therefore, the imagematching degree indicates the estimation accuracy.

Fig.4(c) (e) present three of the test synthetic images. The red contour is the sphere model's linear array image related to the initial guess of the motion parameters, while the green contour corresponds to the optimal solution obtained through the optimization algorithm. The test results show that the initial guess only achieves a rough matching, while the optimal results are closely matched with the ground truth.

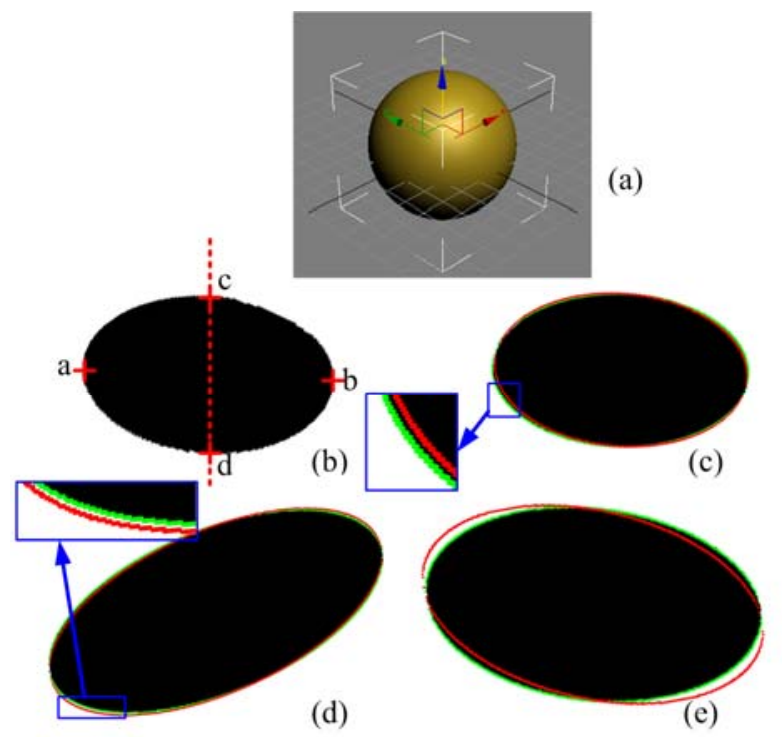

Fig.4. (a) The digital ball; (b) key points picked in the parameter estimation of initial guess; (c) (e) the estimated results of different synthetic images related to different motion parameters: initial guess (red contour) and optimal result (green contour).

The speed (magnitude of the velocity vector) of the ball is the main parameter that we care about. The accuracy of the motion estimation is affected mainly by the ball size, moving speed and camera scanning frequency. Many synthetic images at different ball and camera parameters have been generated and the control variety method has been used for an experimental analysis of the error caused by different factors. 

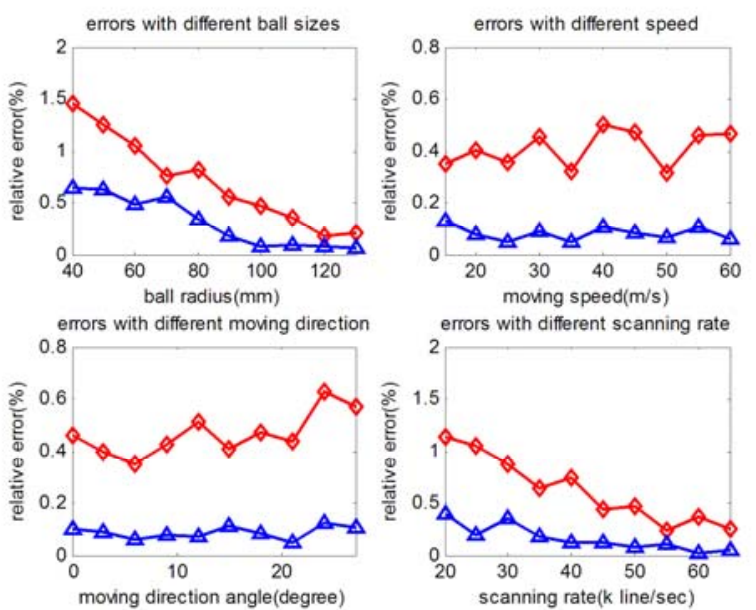

$\checkmark$ Relative error of initial guess

Relative error of optimal solution

Fig.5. Synthetic data tests: the relative errors of the estimated speed (initial guess and optimal solution) with different factors changing.

The relative errors of both initial and optimal speed estimation results are shown in Fig.5. The relative errors are computed by comparing the estimation results with the preset theoretical values. Fig.5 presents the relative errors of the estimated speed with the ball size, ball speed, moving direction and camera scanning rate changing, respectively. When one factor is changing, the other factors are kept unchanged.

From the error graphs, we found that, firstly, due to the optimization algorithm, the accuracy and robustness of the optimal solution of estimation was distinctly refined compared with that of the initial guess. Secondly, when the other factors were unchanged, the speed estimation accuracy was improving with the ball size increasing. We obtained the same results when the camera scanning rate was increasing. Since larger ball size and faster scanning rate provide larger projection contour of the moving ball, more contour information could be used to provide a more accurate estimation. Additionally, the relative errors of the speed estimation do not change significantly when the ball's moving speed and direction are changing. This implies that the velocity of the moving ball does not have a significant impact on the estimation error.

\section{B. Tests on Real Data}

The proposed method has also been tested on the real images in laboratory. The real images are collected using BASLER spL2048-70km line-scan camera (Fig.6(a)). The camera is fixed on a Pan-tilt control instrument. The image plane of the camera can be rotated around the primary optical axis from $-15^{\circ} \sim 15^{\circ}$ manually. The tennis ball is arranged on the platform of the linear guide rail, which is driven by computer and can move at a certain constant speed. The constant speed is regarded as ground truth in real data tests. The relative errors are computed by comparing the estimated speed with the preset constant speed.

A calibration procedure has been performed to acquire camera parameters (including the focus length, lens distortion, camera position and attitude). The camera's attitude was adjusted to make the ball move across the viewing plane of the camera at a right angle.

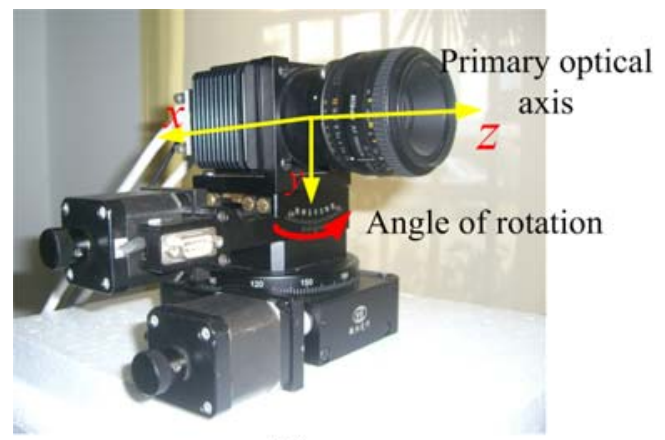

(a)

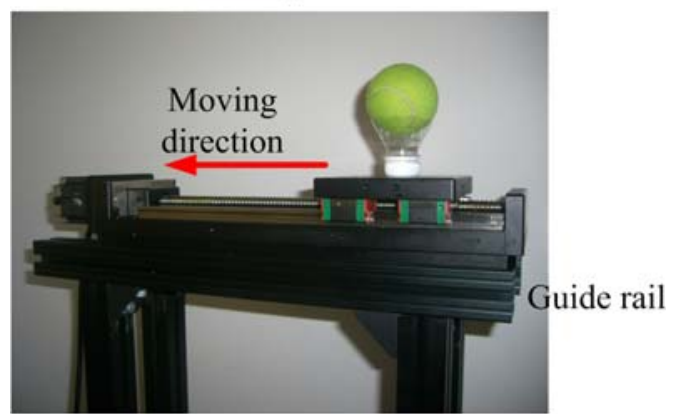

(b)

Fig.6. (a) BASLER spL2048-70km line-scan camera and Pan-tilt control instrument; (b) moving tennis ball on the guide rail.
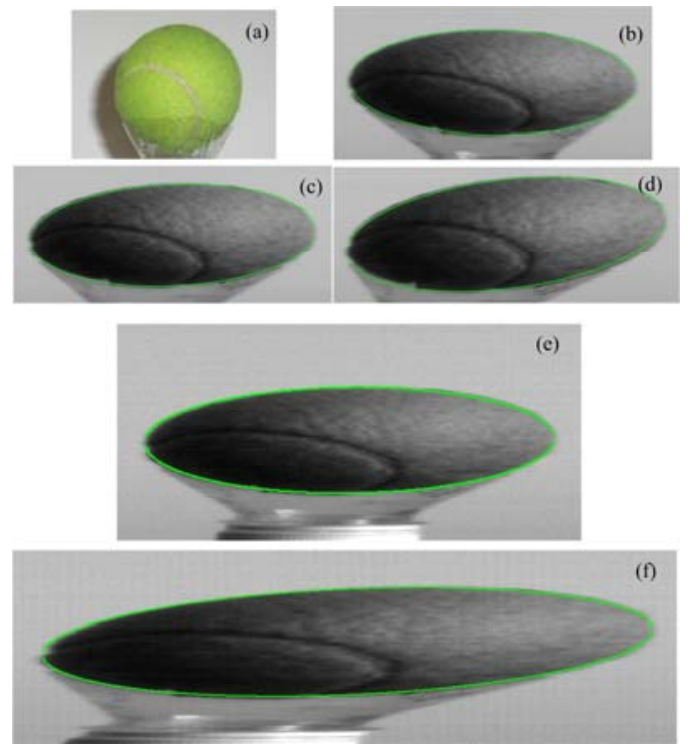

Fig.7. (a) The tennis ball; (b) (f) the estimation results of tennis ball with different moving directions and camera scanning rate (green contour: the projection of the sphere model at the optimal solution of the motion parameters ).

The real ball data are collected as follows. Firstly, the camera scanning rate is kept unchanged, and the Pan-tilt control instrument is adjusted in order to make the viewing plane of the camera rotate in different angles. The adjustment can achieve an equivalent changing of the ball's moving direction. Therefore, the linear array images of the ball 
moving at different moving directions can be collected. Fig.7(b) (d) show three of these ball images, which are collected by rotating angles of $0^{\circ}, 4^{\circ}, 10^{\circ}$, respectively.

Secondly, keep the ball move across the viewing plane perpendicularly, and adjust the camera scanning rate. Therefore, the linear array images of the ball can be collected with different camera scanning rate. Fig.7(e) and (f) show two of these images, which are collected at scanning rate of 100 line/sec, 200 line/sec, respectively.

The proposed method is adapted to deal with all of these ball images. In order to graphically depict the estimation results, we draw the projection contours of the sphere model simulated by the optimal solution of the motion parameters on the original ball images. The simulated green contours are well matched with the ball contours, which mean that the method is able to achieve quite good estimation.

As illustrated in Fig.8, the relative errors of the speed estimation are also computed with the ball moving direction and camera scanning rate changing. From the error graphs, we can also find that the ball moving direction has little impact on the estimation errors. However, the improvement of the camera scanning rate would significantly reduce the estimation errors. This is because in the same time interval, higher scanning rate would make the camera record more spatial information of the moving ball, which helps to improve the effectiveness of the method.
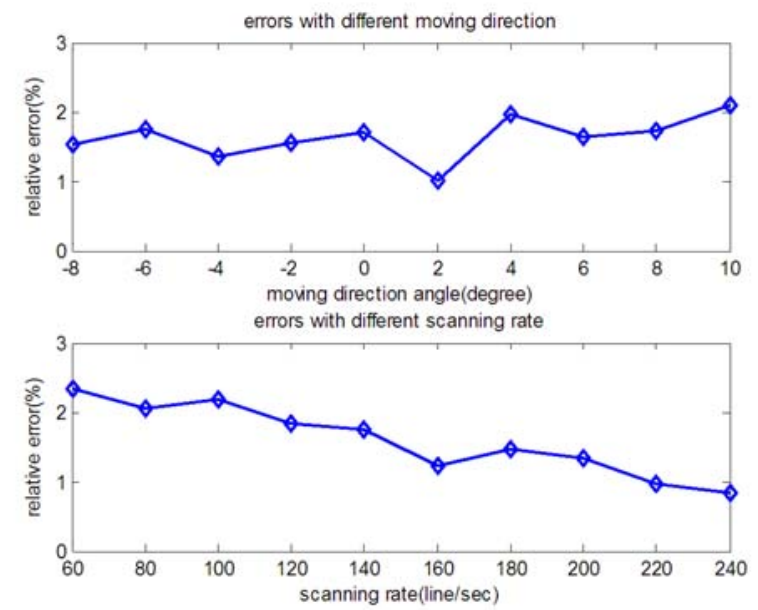

Fig.8. Real ball data tests: the relative errors of the estimated speed (optimal solution) with different factors changing.

\section{CONCLUSION}

In this paper, we have presented a new cost-effective technique for estimating the 3D motion of spherical objects using a single line-scan camera. Cameras have played important roles in measurement [1, 3, 13]. The line-scan camera has its distinctive advantages, such as low data transmission, high scanning frequency and uniform image background, which make it especially suitable for the imaging of a high-speed object. The work presented in this paper shows the valuable potential of linear array image for motion estimation. Compared with the area array image, the linear array can record the time and space information of the moving object in line-scan mode. Moreover, it can acquire a sharp contour of fast-moving object on clear background.

Considering the characteristics of the spherical object, several key points are picked to compute the initial guess of the motion parameters, which is then used to begin a modelbased optimization algorithm. The optimization algorithm achieves a refinement of the initial values by considering the whole contour information of the moving ball, which distinctly improves the effectiveness and robustness of the motion estimation. The analysis about the experimental results could reach a conclusion that the proposed method has little limitation in the ball's motion, and moreover, the improvement of the scanning rate of the camera would further refine the estimation accuracy.

The model-based optimization method transforms the motion estimation into a problem of image-matching, which could be a general scheme for dealing with moving objects. In future research, the efficiency of the optimization should be further improved. Additionally, the method is not only confined to the measurement of a moving ball, but would be further expanded for dealing with common shape objects.

\section{ACKNOWLEDGMENT}

The work was supported by the National Natural Science Foundation of China under Grant No. 60872153. The authors are also grateful for the help of the colleagues at ATR Key Laboratory.

\section{REFERENCES}

[1] Ren, J., Orwell, J., Jones, G.A., Xu, M. (2009). Tracking the soccer ball using multiple fixed cameras. Computer Vision and Image Understanding, 113 (5), 633-642.

[2] Boracchi, G., Caglioti, V., Giusti, A. (2008). Single image 3D reconstruction of ball velocity and spin from motion blur. In The 3rd International Conference on Computer Vision Theory and Applications, 22 January 2008. Funchal, Madeira, Portugal: INSTICC, 22-29.

[3] Caglioti, V., Giusti, A. (2009). Recovering ball motion from a single motion-blurred image. Computer Vision and Image Understanding, 113 (5), 590-597.

[4] Boracchi, G., Caglioti, V., Giusti, A. (2007). Ball position and motion reconstruction from blur in a single perspective image. In The 14th International Conference on Image Analysis and Processing, 10-13 September 2007. Modena, Italy: Univ. of Modena and Reggio Emilia, 87-92.

[5] Caglioti, V., Giusti, A. (2010). On the apparent transparency of a motion blurred object. International Journal of Computer Vision, 86 (2), 243-255.

[6] Lim, M., Limb, J. (2008). Visual measurement of pile movements for the foundation work using a highspeed line-scan camera. Pattern Recognition, 41 (6), 2025-2033.

[7] Nayyerloo, M., Chen, X.Q. et al. (2010). Seismic structural displacement measurement using a highspeed line-scan camera: experimental validation. In New Zealand Society for Earthquake Engineering 2010 Conference, 26-28 March 2010. Wellington, New Zealand: NZSEE, 26-28. 
[8] Ai, L., Yuan, F., Ding, Z. (2008). Measurement of spatial object's exterior attitude based on linear CCD. Chinese Optics Letters, 6 (7), 505-509.

[9] Ji, E.B., Jin, W.K., Hang, J.K., Hyun, S.P. (2009). Estimation of 3-D trajectory of the golf ball using dual linear sensor. In The Fifth International Joint Conference on INC, IMS and IDC, 25-27 August 2009. Seoul, Korea: Institute of Electrical and Electronics Engineers, 2024-2030.

[10] Luna, C.A., Mazo, M. et al. (2010). Calibration of line-scan cameras. IEEE Transactions on Instrumentation and Measurement, 59 (8), 2185-2190.
[11] Horaud, R., Mohr, R., Lorecki, B. (1993). On singlescanline camera calibration. IEEE Transactions on Robotics and Automation, 9 (1), 71-75.

[12] Zhao, Z., Zhang, X. et al. (2011). The estimation for position and velocity of moving ball from single linear array image. In International Conference on Soft Magnetic Materials, 23-24 May 2011. Male, Maldives: IITA, 183-187.

[13] Chen, J.Y. et al. (2010). Development and implementation of a Simplified tool measuring system. Measurement Science Review, 10 (4), 142-146.

Received September 14, 2011. Accepted December 5, 2011. 